\title{
Gravity in higher codimension de Sitter Brane Worlds
}

\author{
I. Olasagasti and K.Tamvakis \\ Physics Department, University of Ioannina, \\ GR-451 10 Ioannina, Greece
}

\begin{abstract}
We study solutions of Einstein's equations corresponding codimension $n>2$ global topological defects with de Sitter slices. We analyze a class of solutions that are cylindrically symmetric and admit positive, negative or zero Bulk cosmological constant. We derive the relevant graviton equations. For an extended brane, the properties of the solution depend on appropriate boundary conditions that the exterior solutions have to satisfy near the core. As an alternative we consider matching copies of the exterior solution related by symmetry. We show that we can get localization only when the Bulk cosmological constant is negative. We obtain a condition on the global defect symmetry breaking scale which ultimately controls the size of the $n-1$ internal dimensions at the position of the brane. The induced metric on the brane, in the case of mirror spacetimes, is a direct product of a de Sitter space and an $(n-1)$-sphere, while the metric of the embedding spacetime is a warped product and the actual size of the $(n-1)$-sphere changes as we move along the radial direction. The solutions possess naked singularities, which nevertheless satisfy no-flow conditions.
\end{abstract}




\section{Introduction}

The idea that the ordinary spacetime can be associated with a Brane embedded in a higher dimensional space has received considerable attention in the last few years[1][2][3][4]. This alternative to Kaluza-Klein compactification assumes the localization of matter degrees of freedom on a topological defect or Brane [5]. Such assumptions are backed by expectations of matter field localization through specific String Theory dynamics for $D$-Branes [6]. In contrast, gravity propagates in the Bulk but it retains its four-dimensional character through an effective localization on the Brane resulting from the curvature of extra dimensions[7][8]. Such is the case of a 3 -Brane in a 5-dimensional $A d S$ spacetime where gravitation on the Brane is Newtonian with corrections small at macroscopic scales. Models with more than one extra dimensions have also been constructed based on higher codimension or transverse dimension defects. Although a number of such solutions are known, arising either from local or global defects, graviton perturbations have only been studied, to the best of our knowledge, in the case of flat 3-Branes[9], [10], [11], [12], [13].

There is a considerable interest in de Sitter space lately, to a large extent motivated by the astrophysical evidence in favor of a present phase of accelerated expansion. Independently of that, there is plenty of motivation to study de-Sitter Branes made up of scalar fields in the Bulk that form a global defect. In this paper we explore a class of codimension $n>2$ spacetimes with non-flat 3-Branes and examine their gravitational excitation spectrum and its localization properties.

Let us consider a $d$-dimensional spacetime composed of a $q$-dimensional Brane embedded in $n$ extra dimensions $(d=n+q)$. The Brane corresponds to a global defect arising from $n$ scalar fields $\phi^{a}$ interacting through a potential

$$
V(\phi)=\frac{\lambda}{4}\left(\phi^{a} \phi^{a}-\eta^{2}\right)^{2}
$$

Such a potential allows for solutions with non-trivial mappings of the vacuum manifold, the $(n-1)$-sphere $\phi^{a} \phi^{a}=\eta^{2}$, onto the $n$-dimensional space transverse to the Brane. The simplest non-trivial configuration is

$$
\phi^{a}=\eta f(r) \frac{y^{a}}{y}
$$

with $y^{2} \equiv y^{a} y^{a}, y^{a}=\left\{y \cos \theta_{1}, y \sin \theta_{1} \cos \theta_{2}, \ldots\right\}$, and $\left\{\theta_{1}, \ldots, \theta_{n-1}\right\}$ angular coordinates on the unit $(n-1)$-sphere. $r$ is a radial coordinate on the space transverse to the defect and for solutions representing a defect the profile of $f(r)$ goes from a vanishing value at the center of the core to 1 at the outside. Defined in this way the core of the defect is the region where the potential is significantly different from zero. This is the region that defines the Brane.

One can quite easily find solutions that solve Einstein's field equations in the region outside the core/Brane, that is, where $f(r)=1$. A complete spacetime solution should also include the solution for the core region, which should me matched to the exterior solutions at 
some matching surface. We consider the outside region and see up to what point this might be sufficient in discussing the localization of gravity. In addition to the energy-momentum tensor for the scalar fields, we include the effect of a Bulk cosmological constant $\Lambda$ whose sign we leave arbitrary. For a class of known cylindrically symmetric solutions for the exterior region we write the graviton equation and find the exact solutions. Ultimately, in order to determine whether gravity is actually localized we need to know the specific form of the core matter content and geometry. Instead of fixing the matter on the core, we proceed by considering the brane as an infinitely thin wall with $n-1$ internal dimensions of finite size. We do this by matching two mirror copies of the exterior solutions. In this picture although standard matter is somehow localized along the transverse direction it can still see the $n-1$ internal dimensions. We thus have two requirements in order to get localized gravity. On the one hand we need to look at the behavior in the direction transverse to the wall, and on the other, just as in standard Kaluza-Klein dimensional reduction, we need the internal dimensions at the wall position to be small enough so that modes wrapping around them are correspondingly massive. This thin wall approach allows us to study the spectrum in more detail, even if we keep our analysis qualitative. For the class of solutions at hand, we find that gravity can be localized only for a negative Bulk cosmological constant and for a certain range of the global defect parameters.

Here is the plan of the paper. In section 2 we present the class of cylindrically symmetric solutions, taken from [14] with appropriate reparametrizations. They correspond to solutions with de Sitter slices and backgrounds with negative, positive and zero Bulk cosmological constant. In section 3 we write the relevant equations for the graviton in warped geometries with a spherically symmetric section. In section 4 we give the radial equation and solve it. We also write the boundary conditions set by the specific nature of the matter making up the core. In section 5 we construct and analyze the mirror spacetimes mentioned above. We explain our conclusions in section 6 .

\section{Cylindrically symmetric Branes}

We begin by writing down the solutions that we are going to take as background solutions. They are represented by cylindrically symmetric metrics of the form

$$
d s^{2}=d r^{2}+e^{2 A(r)}\left(L^{2} d \Omega_{n-1}^{2}+\hat{g}_{\mu \nu} d x^{\mu} d x^{\nu}\right)
$$

where $r$ is the radial coordinate in the space transverse to the Brane and $d \Omega_{n-1}^{2}$ is the line-element for a unit radius $(n-1)$-dimensional sphere. We shall take the metric $\hat{g}_{\mu \nu}$ on the Brane to be that of an $q$-dimensional de Sitter space. The curvature of this de Sitter space will be parametrized in the usual way as $\hat{R}_{\mu \nu}=(q-1) H^{2} \hat{g}_{\mu \nu}$ and $\hat{R}=q(q-1) H^{2}$. The associated energy-momentum tensor is

$$
T_{r}^{r}=-\left(\frac{(n-1) \eta^{2}}{2 L^{2}} e^{-2 A}+\Lambda\right), \quad T_{\mu}^{\nu}=\delta_{\mu}^{\nu} T_{r}^{r}, \quad T_{j}^{i}=-\delta_{j}^{i}\left(\frac{(n-3) \eta^{2}}{2 L^{2}} e^{-2 A}+\Lambda\right)
$$


The following solutions exist [14]

$$
e^{2 A(r)}=\left\{\begin{array}{cl}
\alpha_{+}^{2} \sin ^{2}\left(\beta_{+}\left(r_{0}-r\right)\right) & (\Lambda>0) \\
\alpha_{0}^{2}\left(r-r_{0}\right)^{2} & (\Lambda=0) \\
\alpha_{-}^{2} \sinh ^{2}\left(\beta_{-}\left(r_{0}-r\right)\right) & (\Lambda<0)
\end{array} \quad L^{2}=\frac{\left(n-2-\kappa^{2} \eta^{2}\right)}{(q-1) H^{2}}\right.
$$

For the first and the third cases we have

$$
\alpha_{ \pm}^{2}=\frac{(q-1)(d-1) H^{2}}{2 \kappa^{2}|\Lambda|}, \quad \beta_{ \pm}^{2}=\frac{2 \kappa^{2}|\Lambda|}{(d-2)(d-1)}
$$

while in the second case

$$
\alpha_{0}^{2}=\frac{(q-1)}{(d-2)} H^{2}
$$

$\kappa^{2} \equiv 8 \pi G$ gives the gravitational coupling on the Bulk.

We note that to be well defined we need $n \geq 3$. At some value of $r$ the above exterior solutions should match the undetermined core solution. Without loss of generality we will take this matching hypersurface to be at $r=0$. We also note that these geometries are characterized by a naked singularity at $r=r_{0}$. At that point the energy-momentum tensor

diverges. This should not come as a surprise since this corresponds to the vanishing of the radius of the $(n-1)$-dimensional spheres.

In the above $H$ is an arbitrary parameter. The effective $q$-dimensional curvature changes along $r$ as

$$
\hat{R}_{e f}=\hat{R} e^{-2 A}=\frac{2 q \kappa^{2} \Lambda}{(d-1) \sin ^{2}\left(\beta_{+}\left(r_{0}-r\right)\right)}, \quad \frac{q(d-2)}{\left(r_{0}-r\right)^{2}}, \quad \frac{2 q \kappa^{2}|\Lambda|}{(d-1) \sinh ^{2}\left(\beta_{-}\left(r_{0}-r\right)\right)}
$$

for $\Lambda>0, \Lambda=0, \Lambda<0$, which clearly does not depend on $H$. However we find it convenient to keep it. We can fix it by demanding that it gives the actual Hubble parameter at a given radial position. For example, by demanding that it gives the actual Hubble parameter at $r=0$ we have

$$
H^{2}=\frac{2 \kappa^{2} \Lambda}{(q-1)(d-1) \sin ^{2}\left(\beta_{+} r_{0}\right)}, \frac{(d-2)}{(q-1) r_{0}^{2}}, \frac{2 \kappa^{2}|\Lambda|}{(q-1)(d-1) \sinh ^{2}\left(\beta_{-} r_{0}\right)}
$$

This is clearly equivalent to normalizing the warp factor so that we have $e^{A}=1$ at $r=0$.

\section{Graviton equation}

When considering gravitational perturbations for a gravity-scalars system like the global defect we have considered, the scalar variations $\delta \phi^{a}$, for a transverse, traceless graviton in the harmonic gauge, decouple[15] from the graviton and, thus, can be ignored. In the 
present section we will derive the equation that corresponds to this traceless graviton for a general warped geometry of the type

$$
d s^{2}=e^{2 A(y)} d \hat{s}^{2}+d \tilde{s}^{2} \equiv e^{2 A(y)} \hat{g}_{\mu \nu} d x^{\mu} d x^{\nu}+\gamma_{a b} d y^{a} d y^{b}
$$

where $d \hat{s}^{2}$ is $q$-dimensional and $d \tilde{s}^{2} n$-dimensional. For such metrics we can write the perturbation modes as a product

$$
h_{\mu \nu}(x, y) \equiv e^{2 A(y)} \phi(y) \epsilon_{\mu \nu}(x)
$$

and one finds that the equation for $\phi$ coincides with the equation for a scalar field, namely,

$$
\tilde{\nabla}^{2} \phi+q \tilde{\nabla} A \tilde{\nabla} \phi+e^{-2 A} m^{2} \phi=0 .
$$

where $m^{2}$ is the mass term of the $q$-dimensional spin-two perturbations and the tildes denote geometrical quantities calculated using the metric $d \tilde{s}^{2}$ of the extra dimensions ${ }^{1}$.

Since the solutions that we want to study are cylindrically symmetric, we should specialize to the class of metrics that can be written as (7) with

$$
d \tilde{s}^{2}=\gamma_{a b} d y^{a} d y^{b}=d r^{2}+R^{2}(r) d \Omega_{n-1}^{2}
$$

where $r$ is a radial coordinate in the space transverse to the brane and $d \Omega_{n-1}^{2}$ defined as in the previous section. Because of the symmetries of the solution it is natural to write $\phi(y)=\psi(r) Z_{\ell}(\Omega)$ where $Z_{\ell}(\Omega)$ are spherical harmonics in $S^{(n-1)}$. The equation they satisfy is

$$
\square Z_{\ell}+\ell(\ell+n-2) Z_{\ell}=0
$$

where $\square$ here is the box operator on $S^{(n-1)}$. Plugging this into (8) we get the following equation for $\psi(r)$

$$
\psi^{\prime \prime}+\psi^{\prime}\left(q A^{\prime}+(n-1) \frac{R^{\prime}}{R}\right)+\left(m^{2} e^{-2 A}-\frac{\ell(\ell+n-2)}{R^{2}}\right) \psi=0
$$

For the particular solutions displayed in the previous section we had $R(r)=L e^{A(r)}$. In this case the equation simplifies even further reducing to

$$
\psi^{\prime \prime}+(d-1) A^{\prime} \psi^{\prime}+\left(m^{2}-\frac{\ell(\ell+n-2)}{L^{2}}\right) e^{-2 A} \psi=0
$$

\footnotetext{
${ }^{1}$ The ordinary-space graviton factor satisfies the equation [16]

$$
-\frac{1}{2} \hat{\nabla}^{2} \epsilon_{\mu \nu}+\left(-H^{2}+\frac{m^{2}}{2}\right) \epsilon_{\mu \nu}=0
$$
}


The normalization of the modes is given by the term [9]

$$
\int e^{(d-3) A} \psi^{2} d r \int L^{(n-1)} Z_{\ell}^{2}(\Omega) d \Omega_{n-1} \int \sqrt{\hat{g}} \hat{g}^{\mu \nu} \epsilon_{\lambda \kappa, \mu} \epsilon_{\lambda \kappa, \nu}
$$

So, the condition for normalizable $q$-dimensional modes, in our choice of metric, reduces to

$$
\int d r e^{(d-3) A(r)} \psi^{2}(r)<\infty
$$

The normalization integral includes the core region where we do not know the solution, and extends up to the limiting singular point $r_{0}$ of the outside region.

The zero modes $\left(m^{2}=\ell=0\right)$ for the solutions displayed in the previous section can be easily derived from the above equation. Imposing the normalization condition for them we see that the integral between $r=0$ and $r=r_{0}$ is finite only for the constant zero mode solution $\psi_{0}(r)=$ const.. The other linearly independent choice gives a divergent contribution to the normalization integral due to its behavior near the singularity at $r=r_{0}$.

In order to study the massive spectrum it is convenient to transform the graviton equation into a Schrodinger type differential equation. This can be achieved by introducing a new variable $z$ and a new graviton function $\chi(z)$ defined through

$$
d z=e^{-A} d r \quad, \quad \chi=e^{(d / 2-1) A} \psi
$$

In the following we will refer to this choice as the conformal gauge. After these transformations the graviton equation takes the Schrodinger form

$$
\left\{-\frac{1}{2} \frac{d^{2}}{d z^{2}}+V(z)\right\} \chi(z)=\frac{m^{2}}{2} \chi(z)
$$

where the potential $V$ is defined in terms of the warp function as

$$
V=-\frac{1}{4}(d-2)\left(\ddot{A}-\frac{1}{2}(d-2) \dot{A}^{2}\right)+\frac{1}{2 L^{2}} \ell(\ell+n-2)
$$

Moreover, for $\chi(z)$ the normalizability condition is the usual one, that is,

$$
\int \chi^{2}(z) d z<\infty
$$

\section{Spectrum}

We can work the transformation to the conformal gauge for each of the solutions presented in the previous section. In the case of positive Bulk cosmological constant $\Lambda>0$ we obtain

$$
e^{2 A}=\frac{\alpha_{+}^{2}}{\cosh ^{2}(\tilde{\alpha} z)}
$$


in terms of $\tilde{\alpha}^{2} \equiv \alpha_{+}^{2} \beta_{+}^{2}=(q-1) H^{2} /(d-2)$. The variable $z$ takes values in the range $z_{0}<z<\infty$, where $z_{0} \equiv z(0)$. At the singularity $r=r_{0}$, the variable $z$ reaches infinity. The potential as a function of $z$ is

$$
V(z)=\frac{1}{2 L^{2}} \ell(\ell+n-2)+\frac{\tilde{\alpha}^{2}}{8}\left\{(d-2)^{2}-\frac{d(d-2)}{\cosh ^{2}(\tilde{\alpha} z)}\right\}
$$

The general solution of the above Schrodinger problem is given in terms of hypergeometric functions as

$$
\chi^{(\mp)}(z)=\cosh (\tilde{\alpha} z)^{(\mp \sqrt{c})}{ }_{2} F_{1}\left[d / 4 \pm \sqrt{c} / 2,(2-d) / 4 \pm \sqrt{c} / 2 ; 1 \pm \sqrt{c} ; 1 / \cosh ^{2}(\tilde{\alpha} z)\right]
$$

with $c$ used as a shorthand for

$$
c=\frac{(d-2)^{2}}{4}+\frac{(d-2) \ell(\ell+n-2)}{\left(n-2-\kappa^{2} \eta^{2}\right)}-\frac{(d-2) m^{2}}{(q-1) H^{2}}
$$

To check normalizability we look at the behavior as $z \rightarrow \infty$

$$
\chi^{( \pm)}(z) \sim e^{ \pm \sqrt{c} \tilde{\alpha} z}
$$

The behavior changes qualitatively at $m=m_{c}$, which is the value that makes $c=0$

$$
m_{c}^{2}=H^{2} \frac{(q-1)}{4}\left\{(d-2)+\frac{4 \ell(\ell+n-2)}{n-2-\kappa^{2} \eta^{2}}\right\}
$$

For $c<0$, or equivalently $m^{2}>m_{c}^{2}$, asymptotically the functions approach plane waves

$$
\chi^{( \pm)}(z) \sim e^{ \pm i \sqrt{|c|} \tilde{\alpha} z}
$$

so both will in principle lead to acceptable continuum modes. For $c>0$, or $0<m^{2}<m_{c}^{2}$, only one type of modes is normalizable, namely,

$$
\chi^{(-)}(z) \sim e^{-\sqrt{c} \tilde{\alpha} z}
$$

These modes correspond to the discrete part of the massive spectrum. The number of discrete modes and their mass eigenvalues will depend on $z_{0}$ and the boundary conditions at that point. Note that for $m^{2}=0, \ell=0$, which corresponds to $c=(d-2)^{2} / 4$, we recover the $\psi_{0}(r)=$ const. zero-mode.

For a vanishing Bulk cosmological constant $\Lambda=0$ the variables are related as

$$
r_{0}-r=r_{0} e^{-\left(z-z_{0}\right) \alpha_{0}}
$$

while the range of $z$ is as in the previous case. Here we arrive at a constant potential

$$
V=\frac{\alpha_{0}^{2}}{8}(d-2)^{2}+\frac{1}{2 L^{2}} \ell(\ell+n-2)
$$


Again, the solutions depend on the parameter $c$ defined above in (21). For $c>0$ we have a continuum of plane wave solutions

$$
\chi_{m}^{( \pm)}(z)=N_{m} e^{ \pm i \alpha_{0} \sqrt{|c|} z}
$$

defined in the half line $z_{0}<z<\infty$. The bounded solutions arising for $c>0$

$$
\chi^{(-)}(z)=N^{(-)} e^{-\alpha_{0} \sqrt{c} z}
$$

are subject to the appropriate boundary conditions at $z_{0}$.

Similarly, in the case of negative Bulk cosmological constant $\Lambda<0$, we have

$$
e^{2 A}=\frac{\alpha_{-}^{2}}{\sinh ^{2}(\tilde{\alpha} z)}
$$

for the same range of parameter $z_{0}<z<\infty$ and using again $\tilde{\alpha}^{2}=\beta_{-}^{2} \alpha_{-}^{2}=(q-1) H^{2} /(d-2)$. It is straightforward to obtain the potential as

$$
V(z)=\frac{1}{2 L^{2}} \ell(\ell+n-2)+\frac{\tilde{\alpha}^{2}}{8}\left\{(d-2)^{2}+\frac{d(d-2)}{\sinh ^{2}(\tilde{\alpha} z)}\right\}
$$

It is clear that for $m^{2}>m_{c}^{2}$ (see eq $(23)$ ), we obtain again the continuum of asymptotic plane waves. The existence of acceptable normalizable modes in the interval $\left[0, m_{c}^{2}\right]$ depends on the boundary conditions to be imposed at $z_{0}$. The exact form of the two linearly independent solutions is given by

$\chi(z)^{(\mp)}=\sinh (\tilde{\alpha} z)^{d / 2} \cosh (\tilde{\alpha} z)^{-(d \pm 2 \sqrt{c}) / 2}{ }_{2} F_{1}\left[d / 4 \pm \sqrt{c} / 2,(d+2) / 4 \pm \sqrt{c} / 2 ; 1 \pm \sqrt{c} ; 1 / \cosh ^{2}(\tilde{\alpha} z)\right]$

with $c$ defined as before.

The discrete part of the spectrum, including the zero mode, in all the above three cases rests on the boundary conditions at $z_{0}$, where the outer solutions should match the core solutions. One expects that a regular solution at the center of the core will be possible only for a discrete set of values of $m^{2}$.

As $z \rightarrow \infty$ we encounter the naked singularity. For acceptable solutions we impose unitary boundary conditions, which amount to a no flow condition into the singularity for conserved quantities. From the invariance due to the symmetries along the brane one obtains the constraint

$$
\lim _{z \rightarrow \infty} e^{(d-2) A(z)} \psi(z) \psi^{\prime}(z)=0
$$

We have $\psi(z)=e^{-(d-2) A(z) / 2} \chi(z)$ and as $z \rightarrow \infty$ for all three cases the discrete modes go as $\psi(z) \propto e^{-(d-2) \alpha z / 2-\alpha \sqrt{c} z}$ so that the above turns into

$$
\lim _{z \rightarrow \infty} e^{-\alpha \sqrt{c} z}=0
$$


so the condition is fulfilled. For the continuum modes on the other hand $(c<0) \psi(z) \propto$ $e^{-(d-2) \alpha z / 2-i \alpha \sqrt{|c|} z}$ so now the left hand side of the condition reads

$$
\lim _{z \rightarrow \infty} e^{-i \alpha \sqrt{|c|} z}
$$

This is a wildly oscillating function and corresponds to a function $\chi$ which is not normalizable in a strict sense. As usual realistic states will be described by a superposition that leads to a normalized function which means that $\chi \rightarrow 0$ as $z \rightarrow \infty$ and therefore the condition will be fulfilled as well. Normalizable modes therefore automatically satisfy the no-flow condition and we do not have to worry about new boundary conditions imposed by the singularity.

One can be more specific about the boundary conditions set by the interior solution on the metric functions outside [17]. Taking into account the symmetry of the solution we can expect the $T_{A B}$ components through the interior to be

$$
T_{r}^{r}=f_{r}(r), \quad T_{\nu}^{\mu}=\delta_{\nu}^{\mu} f_{0}(r), \quad T_{\theta^{\prime}}^{\theta}=\delta_{\theta^{\prime}}^{\theta} f_{\theta}(r)
$$

The exterior solution extends from $r=0$, the matching hypersurface, to the singularity at $r=r_{0}$. The core region, on the other hand extends from $r=-r_{c}$ which gives the center of the solution, to the hypersurface $r=0$. If we integrate Einstein's equations, in the form $R_{b}^{a}=\kappa^{2}\left[T_{b}^{a}-\delta_{b}^{a} T /(d-2)\right]$, through the core region $-r_{c}<r<0$ we obtain

$$
\begin{aligned}
\left.R^{\prime} R^{n-2} e^{q A}\right|_{-r_{c}} ^{0}= & \frac{\kappa^{2}}{d-2}\left[\mu_{r}+q \mu_{0}-(q-1) \mu_{\theta}\right]+(n-2) \int_{-r_{c}}^{0} R^{n-3} e^{q A} d \rho \\
\left.A^{\prime} e^{q A} R^{n-1}\right|_{-r_{c}} ^{0}= & \frac{\kappa^{2}}{d-2}\left[\mu_{r}-(n-2) \mu_{0}+(n-1) \mu_{\theta}\right] \\
& +\left(n-2-\kappa^{2} \eta^{2}\right) \int_{-r_{c}}^{0} R^{n-1} e^{(q-2) A} d \rho
\end{aligned}
$$

where

$$
\mu_{i} \equiv \int_{-r_{c}}^{0} R^{n-1} e^{q A} f_{i}(\rho) d \rho
$$

and $i=(r, 0, \theta)$

To have a regular solution at the center, $r=-r_{c}$, we will have the following conditions

$$
R^{\prime}\left(-r_{c}\right)=1, \quad A^{\prime}\left(-r_{c}\right)=A\left(-r_{c}\right)=0
$$

These can be used in (37),(38) so that the left hand side will give the appropriate boundary conditions at $r=0$.

Above we have given exact solutions for the modes in the outside region. To determine the shape of gravity in the brane it is important to know the number and masses of the discrete modes and this in turn requires knowledge about the detailed nature of the core. Instead of choosing a possible core matter we prefer to study an alternative set-up, where we consider an infinitely thin wall at $z=0$ matching two mirror copies of the same spacetime. This is what we will call the mirror spacetimes. 


\section{Mirror spacetimes}

Another way to model branes with higher codimension is to consider an infinitely thin wall just as in the codimension one case. The difference being that the wall will have the topology $\mathcal{M}_{q} \times S^{n-1}$. The infinitely thin wall is thus a factorized space unlike the embedding spacetime which is not generally factorizable this way. In the present section we shall consider matching two copies of the above manifolds across the $\mathcal{M}_{q} \times S^{n-1}$ wall (for simplicity, we are assuming $Z_{2}$ symmetry across the wall).

It is well known that for an infinitely thin wall the metric is continuous across the wall but that the extrinsic curvature is discontinuous. We can characterize this discontinuity by the jump of the extrinsic curvature across the wall, which is related to the localized energy momentum tensor through

$$
[K]_{j}^{i}-\delta_{j}^{i} \operatorname{Tr}[K]=-\kappa^{2} S_{j}^{i}
$$

where $S_{i j}$ is the localized energy momentum tensor, $K_{i j}$ the extrinsic curvature and the brackets denote the difference of a quantity at the two sides of the wall.

We can consider the present set-up as obtained from the one in the previous sections by substituting what we referred to as the interior region by a mirror copy of the exterior solution. Consequently the former smooth matching surface turns into a brane with an induced localized energy-momentum tensor.

For the metrics at hand (7),(9) but in the conformal gauge eq(14), the explicit expression for the extrinsic curvature reads

$$
K_{\nu}^{\mu}=\delta_{\nu}^{\mu} A_{w}^{\prime} e^{-A_{w}} \equiv \delta_{\nu}^{\mu} K_{0}, \quad K_{\beta}^{\alpha}=\delta_{\beta}^{\alpha} \frac{R_{w}^{\prime}}{R} e^{-A_{w}} \equiv \delta_{\beta}^{\alpha} K_{\theta} .
$$

where primes denote derivatives with respect to the conformal radial coordinate $z$. We will assume that

$$
S_{\nu}^{\mu}=\delta_{\nu}^{\mu} S_{0} \quad S_{\beta}^{\alpha}=\delta_{\beta}^{\alpha} S_{\theta}
$$

which is consistent with the symmetry of our metric ansatz. From this we have that

$$
\begin{aligned}
\kappa^{2} S_{0} & =\left[(q-1) K_{0}+(n-1) K_{\theta}\right] \\
\kappa^{2} S_{\theta} & =\left[q K_{0}+(n-2) K_{\theta}\right]
\end{aligned}
$$

A vacuum wall of tension $\sigma$ is characterized by a localized energy-momentum tensor that is proportional to $h_{i j}$, the induced metric on the wall, so that $S_{i j}=-\sigma h_{i j}$. We can see from the above that this relation can only be satisfied if $K_{0}=K_{\theta}$. Such vacuum walls will not be obtained in general warped spacetimes with different warp factors for the $q$-dimensional and $(n-1)$-sphere parts. In that case the wall will have a non-uniform tension, different for $x^{\mu}$ and the angular directions. This is an interesting case to investigate since, unlike the solutions that we are treating in this paper, they are not conformal to factorized spacetimes. We expect to investigate this issue in the future.

Let us stay with the conformally factorized spacetimes. If we consider the solutions used earlier in the paper, we will indeed obtain a vacuum wall when we match two copies 
of the spacetime. Since in order to represent a positive tension wall we will need the warp factors to decrease away from the wall, we can paste two mirror copies of the solutions for $z$ in the range $z_{0}<z<\infty$ matching along the hypersurface given by $z=z_{0}(r=0$ in the original coordinates).

The tension $\sigma$ of this wall will be given by

$$
\kappa^{2} \sigma=-(d-2) e^{-A\left(z_{0}\right)} A^{\prime}\left(z_{0}\right)
$$

As was the case above, we will encounter naked singularities at a finite distance from the brane.

When we go on to study the graviton perturbations, the effective potential at either side of the wall will be mirror copies of the potentials obtained above and in addition we will have a term proportional to a $\delta$ function, which is the fingerprint of the discontinuity introduced by the wall

$$
-\chi^{\prime \prime}+\left[V_{r}+\left(\frac{(q-1)}{2}\left[A^{\prime}\right]+\frac{(n-1)}{2} \frac{\left[R^{\prime}\right]}{R_{0}}\right) \delta\left(z-z_{0}\right)\right] \chi=m^{2} \chi
$$

where $V_{r}$ is the regular part of the potential, which has been calculated above for each of the spacetimes.
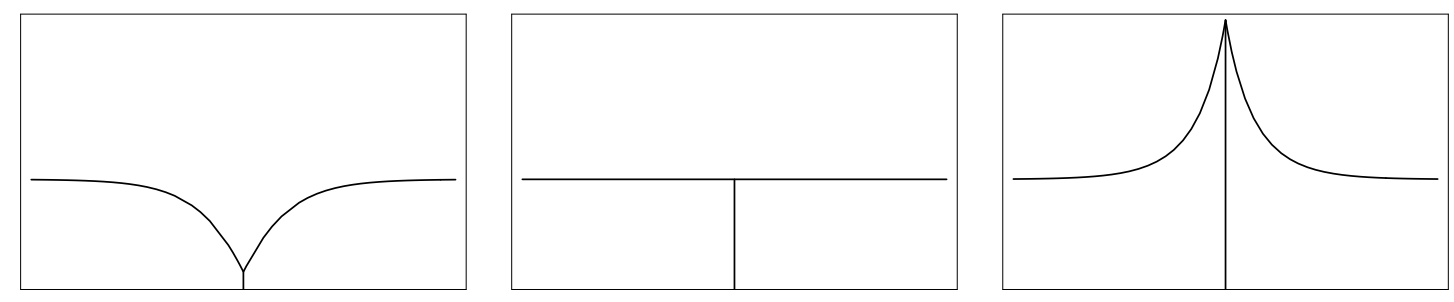

Figure 1: Thin wall potentials for $\Lambda>0, \Lambda=0$ and $\Lambda<0$ respectively.

The delta function gives a boundary condition at the position of the wall. In order to satisfy the above equation, $\chi$ must be continuous but its derivative must have a discontinuity. The jump of the derivative across the wall is given by

$$
\frac{\left[\chi^{\prime}\right]}{\chi_{0}}=\frac{(q-1)}{2}\left[A^{\prime}\right]+\frac{(n-1)}{2} \frac{\left[R^{\prime}\right]}{R_{0}}=\frac{(d-2)}{2}\left[A^{\prime}\right]
$$

where the last equality is valid only for the present conformally factorizable spacetimes. The boundary condition is also satisfied by modes with $\chi_{0}=0,\left[\chi^{\prime}\right]=0$, however these are no concern to us since they are zero at the position of the brane and thus do not couple to matter sitting there.

Eq (48) gives an explicit boundary condition at the wall. As we will show below, it is trivially satisfied by the zero mode $\ell=0, m=0$ in all three cases $(\Lambda>0, \Lambda=0, \Lambda<0)$. 
In Fig.(1) we can see the form that the thin-wall potentials take for each sign of the Bulk cosmological constant.

For $\Lambda>0$ the boundary condition (48) is

$$
\frac{\chi_{0}^{\prime}}{\chi_{0}}=-\frac{d-2}{2} \tilde{\alpha} \tanh \left(\tilde{\alpha} z_{0}\right)
$$

As we showed earlier in the paper the discrete and continuum parts of the spectrum are separated by $c=0$. We showed that when $c>0$ only one of the modes is normalizable, if we now impose the boundary condition set by the wall, only some values of $c$ will be allowed, thus leading to the discrete spectrum. As is apparent from Fig.(1), we can expect a number of discrete modes for different values of $c$.
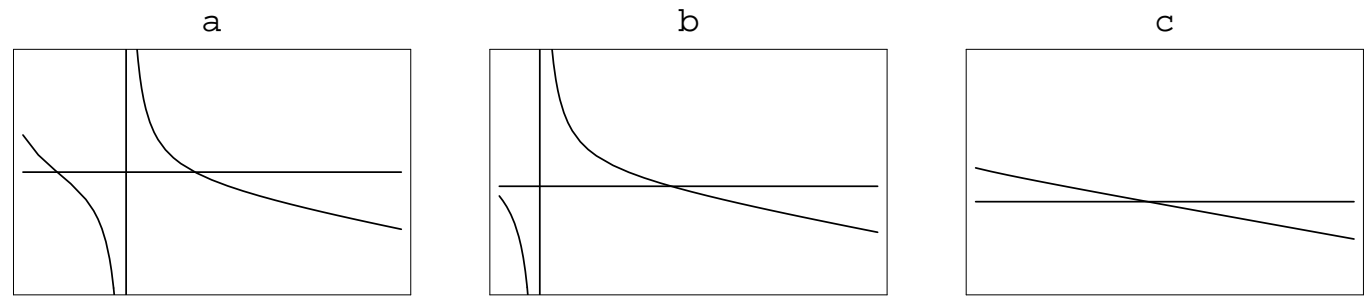

Figure 2: boundary condition equation for $\Lambda>0$. The depth of the well decreases from left to right

In Fig.(2) we represent both sides of equation (49) as a function of $c$ for three extra dimensions $(d=7)$ and three different values of the wall tension. We can see that there is a critical value of the tension $\sigma_{+}$such that for $\sigma<\sigma_{+}$there are two values of $c_{s}$ (with $s$ labelling the values) that satisfy the boundary condition. These lead to two towers of states if we allow for $\ell \neq 0$ modes

$$
m^{2}(\ell, s)=H^{2} \frac{(q-1)}{4}\left\{(d-2)-\frac{4 c_{s}}{(d-2)}+\frac{4 \ell(\ell+n-2)}{n-2-\kappa^{2} \eta^{2}}\right\}
$$

where $c_{1}=(d-2)^{2} / 4$ corresponds to the lowest lying value which leads to

$$
m^{2}(\ell, s=1)=H^{2} \frac{(q-1) \ell(\ell+n-2)}{n-2-\kappa^{2} \eta^{2}}
$$

these modes include the zero mode, $\ell=0$, and then modes with masses $m^{2} \sim H^{2} /\left(n-2-\kappa^{2} \eta^{2}\right)$ which will become more and more massive as $\kappa^{2} \eta^{2} \rightarrow n-2$.

For $\Lambda=0$ the boundary condition (48) is

$$
\frac{\chi_{0}^{\prime}}{\chi_{0}}=-\frac{d-2}{2} \tilde{\alpha}
$$

and only the $c_{1}$ value satisfies the boundary condition for $m^{2}<m_{c}^{2}$. 
For $\Lambda<0$ the boundary condition (48) is

$$
\frac{\chi_{0}^{\prime}}{\chi_{0}}=-\frac{d-2}{2} \tilde{\alpha} \operatorname{coth}\left(\tilde{\alpha} z_{0}\right)
$$

Again only the modes with $c=c_{1}$ satisfy the condition when $m^{2}<m_{c}^{2}$.

What do we get from the above?. As is well known, because we have a de Sitter brane we have a mass gap for the continuum spectrum. The continuum starts at $m=m_{c}$ which is of the order of $H$ for $\ell=0$. We recall that $H$ is the actual Hubble parameter at the wall and is given by eq (6). From the $q$-dimensional perspective the lightest of these modes will be suppressed by their mass only on superhorizon length scales $L>1 / m_{c} \sim 1 / H$. This means that unless their wave functions are suppressed on the brane these modes will lead to higher dimensional gravity. As we see in Fig.(1) there is no suppression for $\Lambda>0$ or $\Lambda=0$ so we can conclude that gravity is not localized in these cases. As is the case in the 5 -dimensional thin wall case, it seems that only the $\Lambda<0$ can do the job.

In this last case we should take care of the modes trapped by the wall with masses given by eq (51). In addition to the zero mode with $\ell=0$, we have all the others with $\ell \neq 0$. It is thus important that these are massive enough, from the $q$-dimensional point of view, so that at observable distances on the brane they can be neglected. This can always be achieved by a symmetry breaking scale $\eta$ sufficiently close to the critical value $\kappa^{2} \eta_{c}^{2} \equiv(n-2)$.

We therefore conclude that in the mirror spacetimes gravity can only be localized when the Bulk cosmological constant is negative. We expect the same to be true for the solutions with a realistic core as well.

\section{Conclusions}

In the present paper we have extended previous analyses on the gravity on braneworld models by considering thick branes embedded in spacetimes with $n \geq 3$ extra dimensions and, most importantly, by allowing a non-flat brane geometry. In particular we have considered a brane geometry with $n-1$ small compactified dimensions and q-dimensional de Sitter slices. We refer to these branes as thick since they do not correspond to a perfectly localized source in the extra dimensions. In the first part we have modelled the branes as a global topological defect associating the brane with the core. The sources in the Bulk are a cosmological constant and the scalar fields that make up the defect. The defect solution is characterized by the fact that at some distance from its center the potential energy reaches a minimum. We refer to this region where the fields have their vacuum values as the exterior region. We have analyzed in some detail the solution in this exterior region with an eye at the localization of the graviton. To this end we have written the relevant equations for the metric perturbations around backgrounds described by warped geometries. We have further specialized the equations for the case at hand, that of spherically symmetric solutions

as seen from the extra dimensions. We have derived the relevant equation for the radial dependence of the perturbations and then applied it to the solutions at hand. 
We have assumed that the exterior solutions match at some boundary hypersurface an unknown interior solution describing the core of the defect, that is, what we consider to be the brane. Focusing on the exterior part of the solution we have found some necessary conditions for gravity to be localized. They are not sufficient because the specific nature of the core will affect the modes through the boundary conditions at the matching surface. The degree of arbitrariness associated with choosing a suitable interior solution describing the defect and matching it to the exterior solution can be circumvented by going to infinitely thin branes. Meaningful and general conclusions can still be drawn in this approach. Thus, in the last part of the paper we have turned our attention to mirror spacetimes. We have taken each of the exterior solutions and we have constructed new solutions by pasting two mirror copies of the same spacetime across a hypersurface, thus creating a thin wall discontinuity which can be associated with the brane. Since we choose a hypersurface orthogonal to the radial direction the resulting wall/brane has topology $M_{q} \times S^{n-1}$.

This is not equivalent to considering the known 5D braneworlds with a number of extra compactified dimensions. This would be the case if the $d$-dimensional solution was a factorized geometry of the form $M_{q+1} \times S^{n-1}$. Instead we have a warped geometry. This means that the size of the $S^{n-1}$ part is not constant throughout the spacetime. This new approach allowed us to solve the problem completely since the presence of the infinitely thin wall fixes the required boundary conditions. For the three signs of the Bulk cosmological constant, the continuum spectrum starts at $m \sim H$. Since $H$ gives the Hubble scale on the wall, these massive modes won't be suppressed on the wall within the horizon and in order to obtain q-dimensional gravity they should couple very weakly to the brane which only happens for $\Lambda$ negative. This is not enough however. The brane in the mirror spacetimes has $n-1$ internal dimensions and there are massive modes that wrap around these whose function along the radial direction is the same as the zero mode and consequently are not suppressed on the brane. They will destroy localized gravity unless they are massive enough so that at observable distances on the brane they can be integrated out. For the solutions considered here the mass of these modes in units of the Hubble parameter mass scale are set by the symmetry breaking scale of the defect. It turns out that they can be made arbitrarily massive by a symmetry breaking scale $\eta^{2}$ that approaches the critical value $(n-2) / \kappa^{2}$. For such values of $\eta$, and always when $\Lambda<0$, we conclude that gravity will be localized.

The solutions analyzed here possess naked singularities. Nevertheless, we have argued that these can be circumvented by imposing no-flow boundary conditions. Indeed, we find that the no-flow condition is automatically satisfied by normalizable modes[18]. The solutions studied here are conformally factorizable and, in that sense, quite simple. We hope to study the gravity of more general solutions, which are not conformally factorizable, in the near future.

\section{Acknowledgements}

The authors acknowledge support from the RTN programme HPRN-CT-2000-00152. They also wish to thank the CERN Theory Division for its hospitality. 


\section{References}

[1] I. Antoniadis, Phys. Lett. B 246(1990)377.

[2] N. Arkani-Hamed, S. Dimopoulos and G. Dvali, Phys. Lett. B 29(1998)263, hepph/9803395.

[3] I. Antoniadis, N. Arkani-Hamed, S. Dimopoulos and G. Dvali, Phys. Lett. B 436(1998), hep-ph/9804398.

[4] L. Randall and R. Sundrum, Phys. Rev. Lett. 83(1999)3370, hep-ph/9905221.

[5] V. A. Rubakov and M. E. Shaposhnikov, Phys. Lett. B 125(1983)136; Phys. Lett. B 125(1983)139.

K. Akama, in Proceedings of the Symposium on Gauge Theories and Gravitation, Nara, Japan, edts. K. Kikkawa, N. Nakanishi and H. Nariai (springer-Verlag, 1983), hepth/0001113.

[6] P. Horava and E. Witten, Nucl. Phys. B 460(1996)460, hep-th/9510209.

[7] L. Randall and R. Sundrum, Phys. Rev. Lett. 83(1999)4690, hep-th/9906064.

[8] M. Gogberashvili, Phys. Lett. A 14(1999)2025, hep-ph/9904383.

[9] S. Randjbar-Daemi and M. Shaposhnikov, Nucl. Phys. B 645 (2002)188, arXiv:hepth/0206016.

[10] C. Csaki, J. Erlich, T. J. Hollowood and Y. Shirman, Nucl. Phys. B 581 (2000) 309 [arXiv:hep-th/0001033].

[11] R. Gregory, Nucl. Phys. B 467 (1996) 159 [arXiv:hep-th/9510202].

[12] C. Charmousis, R. Emparan and R. Gregory, JHEP 0105 (2001) 026 [arXiv:hepth/0101198].

[13] K. Benson and I. Cho, Phys. Rev. D 64, 065026 (2001) [arXiv:hep-th/0104067].

[14] I. Olasagasti and A. Vilenkin, Phys. Rev. D 62, 044014 (2000) [hep-th/0003300].

[15] O. DeWolfe, D. Z. Freedman, S. S. Gubser and A. Karch, Phys. Rev. D 62 (2000) 046008 [arXiv:hep-th/9909134].

[16] J. Garriga and T. Tanaka, Phys. Rev. Lett. 84 (2000) 2778 [arXiv:hep-th/9911055].

[17] T. Gherghetta, E. Roessl and M. E. Shaposhnikov, localize gravity," Phys. Lett. B 491 (2000) 353 [arXiv:hep-th/0006251].

[18] P. Kanti, I. Olasagasti and K. Tamvakis, Phys. Rev. D 66 (2002) 104026, [arXiv:hepth/0207084] 